Pacific Journal of Mathematic 


\title{
MORE SUM THEOREMS FOR TOPOLOGICAL SPACES
}

\author{
Shashi Prabha Arya and M. K. Singal
}

By a sum theorem for topological spaces is meant a theorem of the following type: If $\mathscr{F}$ is a cover of a space $X$, each element of which possesses a property $\mathscr{P}$, then $X$ also possesses the property $\mathscr{P}$. Different types of sum theorems for various classes of topological spaces have been obtained from time to time by various authors. Perhaps the simplest known sum theorem is the locally finite sum theorem which states the following:

If $\left\{F_{\alpha}: \alpha \in \Lambda\right\}$ be a locally finite closed covering of a space $X$ such that each $F_{\alpha}$ possesses a property $\mathscr{P}$, then $X$ possesses $\mathscr{P}$.

The locally finite sum theorem is shown to hold for a large number of important topological properties.

R. E. Hodel [7] obtained three sum theorems which were applicable to all those properties for which the locally finite sum theorem is true and which are closed hereditary (A property is said to be closed hereditary if when possessed by the space, it is also shared by every closed subspace.) Two of these three theorems of Hodel were improved by the authors in [15]. As applications of these theorems, the authors showed that these theorems not only offered new results for many important properties but also improved results of $\mathrm{H}$. Tamano [20, Theorem 2], Y. Katuta [8, Theorem 5], S. Hanai and A. Okuyama [5, Theorem 3] and A. H. Stone [19, Theorem 3]. In the present paper, some more sum theorems are presented. As an application of Theorem 1, a result of A. H. Stone [19, Theorem 2(ii)] is improved. Theorem 2 is a slight improvement of Theorem 3 obtained by the authors in [15]. Applications of Theorem 3 add substantially to the list of properties for which the locally finite sum theorem is true. In Theorem 4, a general technique for proving the locally finite sum theorem for topological properties has been developed. As a consequence, the locally finite sum theorem has been shown to hold for many more properties. Also, it provides a simple and neat proof of the locally finite sum theorem for many properties for which it is known to hold already.

Suppose, for the first two theorems, that $\mathscr{P}$ is a property for which the locally finite sum theorem holds and which is closed hereditary.

THEOREM 1. If $X$ is a regular space and $\left\{V_{\alpha}: \alpha \in \Lambda\right\}$ is a locally 
finite open covering of $X$ such that each $V_{\alpha}$ possesses the property $\mathscr{P}$ and $f r V_{\alpha}$ (frontier of $V_{\alpha}$ ) is Lindelöf for each $\alpha \in \Lambda$, then $X$ possesses the property $\mathscr{P}$.

Proof. In view of Theorem 2 in [15], it is sufficient to prove that each cl $V_{\alpha}$ possesses the property $\mathscr{P}$. Let $\alpha \in \Lambda$ be fixed. Since fr $V_{\alpha}$ is Lindelöf, there exists a countable subfamily $\left\{V_{\alpha_{i}}: i=1,2, \cdots\right\}$ such that $\operatorname{fr} V_{\alpha} \subset \bigcup_{i=1}^{\infty} V_{\alpha_{i}}$. Let $V_{1}=f r V_{\alpha} \sim \bigcup_{i=2}^{\infty} V_{\alpha_{i}}$. Then $V_{1}$ is a closed subset $f r V_{\alpha}$.

$V_{1}$ and $f r V_{\alpha_{1}}$ are disjoint closed Lindelöf subsets of $\mathrm{X}$. By a simple modification of Tychonoff's construction for separating a pair of disjoint closed Lindelöf subsets of a regular space, it is easy to obtain an open set $G_{1}$ such that $V_{1} \subset G_{1} \subset \mathrm{cl} G_{1} \subset V_{\alpha_{1}}$.

Suppose now that for each $i=1,2, \cdots, n-1$, we have defined the sets $V_{i}$ and $G_{i}$ such that $V_{i} \subset G_{i} \subset \operatorname{cl~} G_{i} \subset V_{\alpha_{i}}$. If $V_{n}=f r V_{\alpha} \sim$ $\left[\left(\bigcup_{k=1}^{n-1} G_{k}\right) \cup\left(\bigcup_{k=n+1}^{\infty} V_{\alpha_{k}}\right)\right]$, then $V_{n} \subset V_{\alpha_{n}}$. Again, since $X$ is regular, there exists an open set $G_{n}$ such that $V_{n} \subset G_{n} \subset \operatorname{cl} G_{n} \cup V_{\alpha_{n}}$. Thus by induction there exists a countable family $\mathbb{B}=\left\{G_{n}: n=1,2, \cdots\right\}$ of open sets which covers $f r V_{\alpha}$ and is such that $\left\{\mathrm{cl} G_{n}: n=1,2, \cdots\right\}$ is locally finite. To prove that (S) covers $f r V_{\alpha}$, let $x \in f r V_{\alpha}$ and let $n$ be the largest integer such that $x \in V_{\alpha_{n}}$. Then $x \in \bigcup_{k=1}^{n} V_{\alpha_{k}}$ and $x \notin \bigcup_{k=n+1}^{\infty} V_{\alpha_{k}}$. If $x \notin \bigcup_{k=1}^{n \rightarrow 1} G_{k}$, then $x \in$ fr $V_{\alpha_{k}} \sim\left[\bigcup_{k=1}^{n-1} G_{\alpha_{k}} \cup\left(\bigcup_{k=n+1}^{\infty} V_{\alpha_{k}}\right)\right]=$ $V_{n} \subset G_{n}$. Also each $\mathrm{cl} G_{n}$, being a closed subset of $V_{\alpha_{n}}$, possesses the property $\mathscr{P}$. If $F_{0}=\operatorname{cl} V_{\alpha} \sim \bigcup_{k=1}^{\infty} G_{k}$, then $F_{0}$ is a closed subset of $\operatorname{cl} V_{\alpha}$ and hence of $X$. Since $f r V_{\alpha} \subset \bigcup_{k=1}^{\infty} G_{k}$, we have, $F_{0} \subset \operatorname{cl} V_{\alpha} \sim$ fr $V_{\alpha}=V_{\alpha}$. It follows that $F_{0}$ possesses the property $\mathscr{P}$. Thus $\left\{\mathrm{cl} G_{n}: n=1,2, \cdots\right\} \cup F_{0}$ is a locally finite closed covering of $\operatorname{cl} V_{\alpha}$, each member of which possesses the property $\mathscr{P}$. It follows that $\mathrm{cl} V_{\alpha}$ possesses $\mathscr{P}$. This completes the proof of the theorem.

Definition 1. [Y. Katuta, 8]. A family $\left\{A_{\alpha}: \alpha \in \Lambda\right\}$ is said to be order locally finite if there is a linear ordering ' $<$ ' of the index set $\Lambda$ such that for each $\alpha \in \Lambda$, the family $\left\{A_{\beta}: \beta<\alpha\right\}$ is locally finite at each point of $A_{\alpha}$.

Every $\sigma$-locally finite family is order locally finite but not conversely.

Definition 2. [Aull, 1]. A subset $A$ of a space $X$ is said to be $\alpha$-paracompact if every open (in $X$ ) covering of $A$ has a locally finite (at points of $X$ ) open (in $X$ ) refinement.

THEOREM 2. Let $X$ be a regular space and let $\mathscr{V}$ be an order locally finite open covering of $X$ such that each $V \in \mathscr{V}$ possesses $\mathscr{P}$ 
and fr $V$ is $\alpha$-paracompact for each $V \in \mathscr{Y}$. Then $X$ possesses $\mathscr{P}$.

Proof. Let $V \in \mathscr{V}$. Since $f r V$ is $\alpha$-paracompact and $X$ is regular, there exists a locally finite open (in $X$ ) covering $\left\{U_{\alpha}: \alpha \in \Lambda\right\}$ of $f r V$ such that each cl $U_{\alpha}$ is contained in some member of $\mathscr{Y}$. For each $\alpha \in \Lambda$, let $W_{\alpha}=\operatorname{cl} U_{\alpha} \cap \operatorname{cl} V$ and let $W_{0}=\operatorname{cl} V \sim \bigcup_{\alpha \in \Lambda} U_{\alpha}$. It follows that $W_{0}$ and each $W_{\alpha}$ possesses the property $\mathscr{P}$. Thus, $\left\{W_{\alpha}: \alpha \in \Lambda\right\} \cup W_{\circ}$ is a locally finite closed covering of cl $V$ every member of which possesses the property $\mathscr{P}$. Hence $X$ possesses $\mathscr{P}$ in view of Theorem 2 in [14].

CoRollary 1 [Singal and Arya, 15]. If of be an order locally finite open covering of a regular space $X$ such that each $V \in \mathscr{Y}$ possesses $\mathscr{P}$ and fr $V$ is compact for each $V \in \mathscr{Y}$, then $X$ possesses $\mathscr{P}$.

We shall now examine those properties of topological spaces to which Theorems 1 and 2 are applicable.

REMARK 1. We list below those properties of topological spaces for which the locally finite sum theorem holds:

Regularity [12], normality, [9], collectionwise normality [9], complete normality [9], perfect normality [9], monotone normality [6], metrizability [12], symmetrizability [3], paracompactness [15], pointwise paracompactness [7], subparacompactness [16], M-paracompactness and normality [14], M-subparacompactness [17], stratifiability [2], the property of being a normal $M$-space [10], the property of being a $\sigma$-space [13], the property of being an aleph space [18], the property of being a locally Lindelöf space [22], the property of being a space of countable type [21], and the property of being a $\Sigma$-space [11].

REMARK 2. The following properties of topological spaces are hereditary:

Regularity, perfect normality, complete normality, metrizability, stratifiability, the property of being a $\sigma$-space, the property of being an aleph space.

REMARK 3. The following properties of topological spaces are closed hereditary:

Normality, collectionwise normality, monotone normality, paracompactness, pointwise paracompactness, subparacompactness, Mparacompactness, $M$-subparacompactness, the property of being a locally Lindelöf space, the property of being a normal $M$-space, the 
property of being a space of countable type.

It follows that Theorems 1 and 2 hold for all properties mentioned in Remarks 2 and 3 above. When applied to metrizability, Theorem 1 improves a result of A. H. Stone [19, Theorem 2(ii)]. Many new results are obtained when Theorems 1 and 2 are applied to other properties of topological spaces listed in Remarks 2 and 3 above.

THEOREM 3. Let $\mathscr{P}$ be a property for which the locally finite sum theorem holds. Then the locally finite sum theorem also holds for the property hereditarily $\mathscr{P}$.

Proof. Let $\left\{F_{\alpha}: \alpha \in \Lambda\right\}$ be a locally finite closed covering of a space $X$ such that each $F_{\alpha}$ possesses the property $\mathscr{P}$ hereditarily. Let $A$ be any subset of $X$. Then $\left\{A \cap F_{\alpha}: \alpha \in \Lambda\right\}$ is a locally finite closed (in $A$ ) covering of $A$. Since each $F_{\alpha}$ possesses the property $\mathscr{P}$ hereditarily, each $A \cap F_{\alpha}$ possesses $\mathscr{P}$. Hence, in view of the hypothesis, $X$ possesses $\mathscr{P}$.

In view of Theorem 2 of R. E. Hodel [7], Theorem 2 of the authors [15] and Theorems 1 and 2 obtained above, we have the following important corollaries to Theorem 3 above for any property $\mathscr{P}$ for which the locally finite sum theorem holds. In the following corollaries $2-5, \mathscr{P}$ is a property for which LFST holds.

CoRollary 2. If $\mathscr{f}$ be a $\sigma$-locally finite elementary covering of a space $X$ such that each $V \in \mathscr{V}$ possesses the property $\mathscr{P}$ hereditarily, then $X$ possesses $\mathscr{P}$ hereditarily.

CoROLlaRY 3. If $\mathscr{Y}$ be an order locally finite open covering of a space $X$ such that for each $V \in \mathscr{Y}$, cl $V$ possesses the property $\mathscr{P}$ hereditarily, then $X$ possesses $\mathscr{P}$ hereditarily.

CoROLlaRY 4. If 9 be a locally finite open covering of a regular space $X$ such that each $V \in \mathscr{V}$ possesses $\mathscr{P}$ hereditarily and fr $V$ is Lindelöf for each $V \in \mathscr{Y}$, then $X$ possesses $\mathscr{P}$ hereditarily.

COROLLARY 5. If $\mathscr{V}$ be an order locally finite open covering of a regular space $X$ such that each $V \in \mathscr{Y}$ possesses the property $\mathscr{P}$ hereditarily and fr $V$ is $\alpha$-paracompact for each $V \in \mathscr{Y}$, then $X$ possesses $\mathscr{P}$ hereditarily.

REMARK 4. In view of Theorem 3 above, it follows that the locally finite sum theorem holds for the property hereditarily $\mathscr{P}$, 
where $\mathscr{P}$ stands for any property mentioned in Remark 1. Also, the above Corollaries 2 to 5 hold for any property $\mathscr{P}$ mentioned in Remark 1.

THeOREM 4. Let $\mathscr{P}$ be a topological property satisfying the following:

(a) The disjoint topological sum of spaces possessing the property $\mathscr{P}$ also possesses $\mathscr{P}$.

(b) $\mathscr{P}$ is preserved under finite-to-one, closed continuous mappings.

Then the locally finite sum theorem holds for $\mathscr{P}$.

Proof. The proof is based on a well-known construction which is essentially due to Morita [10, p. 871]. Let $\left\{F_{\alpha}: \alpha \in \Lambda\right\}$ be a locally finite closed covering of $X$ such that each $F_{\alpha}$ possesses the property $\mathscr{P}$. For each $\alpha \in \Lambda$, let $K_{\alpha}$ denote a copy of $F_{\alpha}$ and let $f_{\alpha}$ be this homeomorphism. Let $X^{*}$ be the disjoint topological sum of $K_{\alpha}$ 's. Let $f: X^{*} \rightarrow X$ be the mapping defined as follows:

For each $x \in X^{*}, f(x)=f_{\alpha}(x)$ if $x \in K_{\alpha}$. In view of the hypothesis (a), $X^{*}$ possesses $\mathscr{P}$. It can be easily verified that $f$ is a finite-toone, closed continuous mapping. It follows, in view of hypothesis (b), that $X$ possesses the property $\mathscr{P}$. This completes the proof of the theorem.

COROLlaRy 6. If $\mathscr{P}$ be a property which is preserved under disjoint topological sums and under perfect maps (quasi-perfect maps, quotient maps or closed continuous maps), then the locally finite sum theorem holds for $\mathscr{P}$.

We shall now examine the preservation of different properties of topological spaces under disjoint sums and under finite-to-one closed continuous maps.

REMARK 5. The disjoint topological sum of spaces possessing the property $\mathscr{P}$ possesses $\mathscr{P}$, where $\mathscr{P}$ stands for any of the following properties:

Regularity, normality, perfect normality, collectionwise normality, complete normality, monotone normality, metrizablity, paracompactness, countable paracompactness, pointwise paracompactness, subparacompactness, stratifiability, semi-stratifiability [4], the property of being a normal $M$-space, local compactness, the property of being a locally Lindelöf space, the property of being a $P$-space (that is, 
every $G_{\tilde{\delta}}$-set is open), the property of being a Hausdorff strongly paracompact and locally Lindelöf space, the property of being a $\sigma$-space, the property of being an aleph space, the property of being a $\Sigma$-space, the property of being a Cech-complete space, the property of being a $k c$-space, the properey of being a space of countable type, local connectedness, local pathwise connectedness.

REMARK 6 . The following properties are preserved under perfect maps:

Regularity, metrizability, local compactness, paracompactness, countable paracompactness, the property of being a Hausdorff strongly paracompact and locally Lindelöf space, the property of being a space of countable type, the property of being a $k c$-space, the property of being a $\sigma$-space, the property of being an aleph space, the property of being a Cech-complete space, the property of being a normal $M$-space.

REMARK 7. The following properties are preserved under closed continuous maps:

Normality, perfect normality, collectionwise normality, complete normality, monotone normality, the property of being a $P$-space, local connectedness, local pathwise connectedness, pointwise paracompactness, subparacompactness, M-subparacompactness, paracompactness and Hausdorff property, stratifiability, semi-stratifiability, the property of being a normal $T_{1}-\sigma$-space.

In view of Remarks 5 to 7, it follows from Theorem 4 and Corollary 6 that the locally finite sum theorem holds for all properties mentioned in Remark 5. Thus, a simple and neat proof of the locally finite sum theorem is obtained for most of the properties mentioned in Remark 1. Also, it follows that the locally finite sum theorem holds also for the following properties of topological spaces besides the ones mentioned in Remark 1:

Local connectedness, local pathwise connectedness, local compactness, countable paracompactness, the property of being a semi-stratifiable space, the property of being a Cech complete space, the property of being a $P$-space, the property of being a $k c$-space.

Leaving aside local connectedness and local pathwise connectedness, all the above properties are closed hereditary (at least!). This means that Theorem 2 of Hodel [7], theorem 2 of the authors [15] and Theorems 1 and 2 of this paper are applicable to all these properties and thus many new results are obtained.

The authors are grateful to the referee for his valuable suggestions. 


\section{REFERENCES}

1. C. E. Aull, Paracompact subsets. General Topology and its Relations to Modern Analysis and Algebra II, Proc. of the Second Prague Symposium, (1966), 45-51.

2. J. G. Ceder, Some generalizations of metric spaces, Pacific J. Math., 11 (1961), 105-125.

3. M. M. Coban, Multivalued mappings and some associated problems, Soviet Math. Dokl., 190 (1970), 105-108.

4. G. D. Creede, Semi-stratifiable spaces, Doctoral Dissertation, Arizona State University, 1968.

5. S. Hanai and A. Okuyama, On paracompactness of topological spaces, Proc. Japan Acad., 36 (1960), 466-469.

6. R. W. Heath, D. J. Lutzer and P. L. Zenor, Monotonically normal spaces, Trans. Amer. Math. Soc., 178 (1973), 481-493.

7. R. E. Hodel, Sum theorems for topological spaces, Pacific J. Math., 30 (1969), 59-65.

8. Y. Katuta, On paracompactness and metrizability of spaces, Proc. Japan Acad., 43 (1967), 748-751.

9. K. Morita, On spaces having the weak topology with respect to closed coverings II, Proc. Japan Acad., 30 (1954), 711-717.

10. - Some properties of M-spaces, Proc. Japan Acad., 43 (1967), 869-872.

11. K. Nagami, $\Sigma$-spaces, Fund. Math., 65 (1969), 169-192.

12. J. Nagata, On a necessary and sufficient condition of metrizability, J. Inst. Polytech. Osaka City University, I (1950), 93-100.

13. A. Okuyama, Some generalizations of metric spaces, their metrization theorems and product spaces, Sci. Rep. Tokyo Kyoiku Daigaku, 9 (1967), 236-254.

14. M. K. Singal and Shashi Prabha Arya, On m-paracompact spaces, Math. Ann. 181 (1969), 119-133.

15. ㄴ Two sum theorems for topological spaces, Israel J. Math., 8 (1970), 155158.

16. M. K. Singal and Pushpa Jain, On Subparacompact and countably subparacompact spaces, Bull Austr. Math. Soc., 5 (1971), 289-304.

17. — M-subparacompact spaces, Jnanabha, 2 (1972), 143-158.

18. — A note on $\aleph$-spaces, Kyungpook Math. J., 13 (1973), 203-210.

19. A. H. Stone, Metrizability of unions of spaces, Proc. Amer. Math. Soc., 10 (1959), 361-366.

20. H. Tamano, Note on paracompactness, J. Math. Kyoto Univ., 3 (1963), 137-143.

21. J. E. Vaughan, Perfect mappings and spaces of countable type, Canad. J. Math. 22 (1970), 1208-1210.

22. Y. Yasui, Some generalizations of $V$. Trnkova's theorem on unions of strongly paracompact spaces, Proc. Japan Acad., 43, (1967), 17-22.

Received May 22, 1973 and in revised form April 26, 1975.

Institute of Advanced Studies, University Meerut

Somerville College, OXford OX2 6HD, U.K

AND

Meerut University, Meerut, India 



\section{PACIFIC JOURNAL OF MATHEMATICS}

\section{EDITORS}

RICHARD ARENS (Managing Editor) University of California

Los Angeles, California 90024

\section{R. A. Beaumont}

University of Washington Seattle, Washington 98105
J. DugundJI Department of Mathematics University of Southern Californıa Los Angeles, California 90007

D. Gilbarg aNd J. Milgram Stanford University Stanford, California 94305

\section{ASSOCIATE EDITORS}

E. F. BeCKENBACH

B. H. NeUmanN

F. Wolf

K. YoSHIDA

\section{SUPPORTING INSTITUTIONS}

UNIVERSITY OF BRITISH COLUMBIA CALIFORNIA INSTITUTE OF TECHNOLOGY UNIVERSITY OF CALIFORNIA MONTANA STATE UNIVERSITY UNIVERSITY OF NEVADA NEW MEXICO STATE UNIVERSITY OREGON STATE UNIVERSITY UNIVERSITY OF OREGON OSAKA UNIVERSITY
UNIVERSITY OF SOUTHERN CALIFORNIA STANFORD UNIVERSITY UNIVERSITY OF TOKYO UNIVERSITY OF UTAH WASHINGTON STATE UNIVERSITY UNIVERSITY OF WASHINGTON AMERICAN MATHEMATICAL SOCIETY NAVAL WEAPONS CENTER 


\section{Pacific Journal of Mathematics \\ Vol. 59, No. 1 \\ May, 1975}

Shashi Prabha Arya and M. K. Singal, More sum theorems for topological

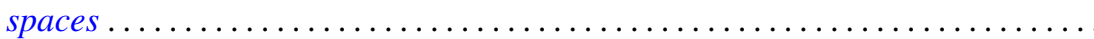

Goro Azumaya, F. Mbuntum and Kalathoor Varadarajan, On M-projective and

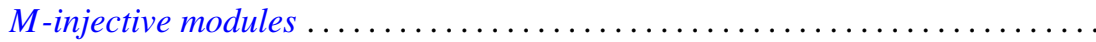

Kong Ming Chong, Spectral inequalities involving the infima and suprema of

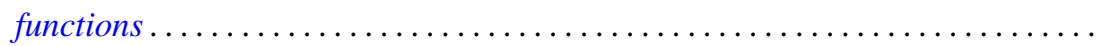

Alan Hetherington Durfee, The characteristic polynomial of the monodromy .......

Emilio Gagliardo and Clifford Alfons Kottman, Fixed points for orientation

preserving homeomorphisms of the plane which interchange two points ......

Raymond F. Gittings, Finite-to-one open maps of generalized metric spaces .......

Andrew M. W. Glass, W. Charles (Wilbur) Holland Jr. and Stephen H. McCleary,

$a^{*}$-closures of completely distributive lattice-ordered groups .............

Matthew Gould, Endomorphism and automorphism structure of direct squares of

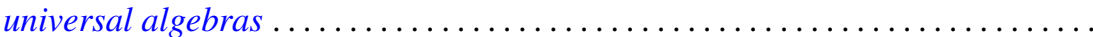

R. E. Harrell and Les Andrew Karlovitz, On tree structures in Banach spaces .....

Julien O. Hennefeld, Finding a maximal subalgebra on which the two Arens

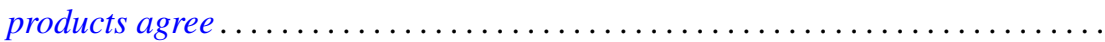

William Francis Keigher, Adjunctions and comonads in differential algebra .......

Robert Bernard Kelman, A Dirichlet-Jordan theorem for dual trigonometric

series

Allan Morton Krall, Stieltjes differential-boundary operators. III. Multivalued operators-linear relations...

Hui-Hsiung Kuo, On Gross differentiation on Banach spaces .

Tom Louton, A theorem on simultaneous observability ...

Kenneth Mandelberg, Amitsur cohomology for certain extensions of rings of algebraic integers.

Coy Lewis May, Automorphisms of compact Klein surfaces with boundary . . .

Peter A. McCoy, Generalized axisymmetric elliptic functions .

211

Muril Lynn Robertson, Concerning Siu's method for solving $y^{\prime}(t)=F(t$, $y(g(t)))$. .

Richard Lewis Roth, On restricting irreducible characters to normal

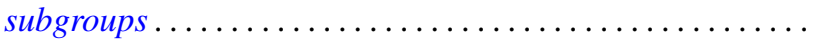

Albert Oscar Shar, $P$-primary decomposition of maps into an $H$-space .

Kenneth Barry Stolarsky, The sum of the distances to certain pointsets on the unit circle.

Bert Alan Taylor, Components of zero sets of analytic functions in $C^{2}$ in the unit ball or polydisc

Michel Valadier, Convex integrands on Souslin locally convex spaces ...

Januario Varela, Fields of automorphisms and derivations of $C$

Arnold Lewis Villone, A class of symmetric differential operators with deficiency

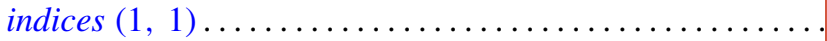

